\title{
Clinical and microbiological effects of systemic ciprofloxacin and metronidazole in Aggregatibacter actinomycetemcomitans-associated periodontitis
}

\author{
Nasrin Esfahanizadeh ${ }^{1}$, Soroosh Khalilinejad ${ }^{1 *}$, Leyla Zonubi ${ }^{2}$ and Hamidreza Daneshparvar \\ ${ }^{1}$ Department of Periodontology, School of Dentistry, Islamic Azad University, Iran. \\ ${ }^{2}$ Department of Microbiology, School of Medical Sciences, Iran University of Medical Sciences, Iran. \\ ${ }^{3}$ Department of Forensic Medicine, Legal Medicine Research Center, Legal Medicine Organization, Tehran, Iran.
}

Received 10 September, 2010; Accepted 7 April, 2014

\begin{abstract}
Destructive periodontal disease is a concern because of the potential damage to the dentition and the financial burden of treatment. It is generally agreed that the microorganisms residing in periodontal pockets are responsible for periodontitis. Approximately $\mathbf{5 0 0}$ bacterial taxa inhabit periodontal pockets. Association between Aggregatibacter actinomycetemcomitans and destructive and progressive forms of periodontitis has been demonstrated by many authors. The aim of this study was to evaluate the microbiological and clinical effects of systemic metronidazole-ciprofloxacin therapy. In this triple-blind controlled clinical randomized trial, 24 patients with at least 4 sites with $\geq 4 \mathrm{~mm}$ of clinical attachment loss and detection of $A$. actinomycetemcomitans were included. The patients were randomly divided into two groups. The patients in the test group received scaling/root planning (S/RP) and ciprofloxacin plus metronidazole, and the patients in the control group received S/RP and placebo representing the aforementioned antibiotics. Bacteria culturing and recording of clinical Att. loss (CAL), gingival index (GI), plaque index (PI) and bleeding on probing (BOP) were done at the baseline, 10 days, 3 and 6 months after the mechanical therapy. There was no significant difference between test and control groups in A. actinomycetemcomitans colony count, CAL, GI, PI and BOP at the baseline. A significant difference was seen in each group between baseline and each of the other research stages for all the recorded parameters. Test group showed a significant difference in A. actinomycetemcomitans colony count, BOP and GI on 3rd and 6th months of research as compared to the baseline. No significant difference was seen between two groups in CAL and PI. Application of ciprofloxacin plus metronidazole as an adjunctive to mechanical therapy has significant effects on periodontal clinical parameters and eradication of $A$. actinomycetemcomitans from periodontal tissues.
\end{abstract}

Key words: Ciprofloxacin, metronidazole, periodontitis, Aggregatibacter actinomycetemcomitans.

\section{INTRODUCTION}

Destructive periodontal disease is a concern because of the potential damage to the dentition and the financial burden of treatment (Slots, 2002). It is well established that microbiological accumulations on the tooth surface cause inflammatory reactions in the adjacent periodontal tissues (Muller et al., 1998). One of these microorganisms 
is Aggregatibacter actinomycetemcomitans, a Gramnegative rod-shaped bacterium (Pavicic et al., 1994). Forms of periodontitis associated with the presence of $A$. actinomycetemcomitans are aggressive periodontitis, sever types of periodontitis and periodontitis as a manifestation of systemic diseases (Marc et al., 2006). Patients suffering from $A$. actinomycetemcomitansassociated periodontitis often respond less favorably to conventional therapy, consisting of mechanical debridement (Pavicic et al., 1994). Renvert et al. (1990) showed that elimination of $A$. actinomycetemcomitans could not predictably be established by repeated mechanical treatment and periodontal surgery in periodontal patients (Renvert et al., 1990). Many other studies showed that $A$. actinomycetemcomitansassociated periodontitis responds poorly to mechanical therapy (Van Winkelhoff and Carolien, 1992). The ability of $A$. actinomycetemcomitans to penetrate epithelial cells and colonize the periodontal tissues around lesion has given support to this pathogen.

Therefore, adjunctive antibiotic has been advised in the treatment of $A$. actinomycetemcomitans-associated periodontitis in an attempt to eradicate the stated microorganism (Karen and Novak, 2006). Thus, the aim of this study was to evaluate the effects of ciprofloxacinmetronidazole therapy as an adjunct to mechanical therapy in treatment of $A$. actinomycetemcomitans-associated periodontitis and eradication of this microorganism.

\section{MATERIALS AND METHODS}

\section{Patients}

A total number of 24 patients with untreated periodontitis and subgingival detection of $A$. actinomycetemcomitans were enrolled into the study. All patients had at least 4 teeth with $4 \mathrm{~mm}$ or above of attachment loss. The patients were enrolled if $A$. actinomycetemcomitans culture and bleeding on probing on these four teeth were positive.

Patients with any of the following conditions were excluded from the study: having any systemic diseases, requirement for antibiotic prophylaxis, receiving any periodontal treatment in the previous 3 months, intake of medications which influence the periodontal tissues (such as non-steroid anti-inflammatory drug (NSAIDS), calcium canal blockers, anticonvulsants, cyclosporine A), allergy against ciprofloxacin or metronidazole, pregnancy or lactation or use of antibiotics in the previous month. All patients enrolled into the study signed the informed consent approved by the Ethics Committee of the Dental Faculty, Azad University.

\section{Clinical measurements}

Probing depth, attachment loss, plaque index, gingival index and bleeding on probing were assessed in all patients. Sub-gingival samples were also prepared from the sites with $4 \mathrm{~mm}$ attachment loss to detect $A$. actinomycetemcomitans.

Probing depth, attachment loss and bleeding on probing were assessed using a William's probe (Hu-friedy, USA). Gingival index assessment was also carried out using the same probe and the criteria demonstrated by Loe and Sillness (1963).

\section{Samples}

The subject's tooth has been isolated by sterile cotton roles and sub-gingival sample were obtained with two sterile paper cones (\#30) penetrated into the deepest site of the periodontal pocket. After $20 \mathrm{~s}$, the paper cones were transferred to the culturing medium, which will be described later, and have been placed in the center of the plate and sent to the laboratory immediately.

\section{Treatment}

All patients that underwent periodontal treatment consisted of suband supra-gingival debridement (using conventional periodontal curettes and ultrasonic scaler) and oral hygiene instruction (modified Bass technique). All the treatment steps were carried out by a well-trained dentistry student under the supervision of a periodontist. After this initial therapy, patients were randomly assigned to receive the antibiotic packs labeled either "1" or "2". One of the numbers was antibiotic and the other was placebo. Because this study was triple-blind, neither the researchers nor the patients were aware of the contents of a pack. The regimen was $250 \mathrm{mg}$ metronidazole, three times daily for a week and $500 \mathrm{mg}$ ciprofloxacin, twice a day for 8 days.

All the measurements and samples collecting were repeated 10 days, 3 and 6 months after the mechanical therapy.

\section{Microbiological analysis}

In this study, the medium used to culture $A$. actinomycetemcomitans was tryptic soy serum plus bacitracin and vancomycin (TSBV). It consists of trypticase soy agar $(40 \mathrm{~g} / \mathrm{L})$ and yeast extract $(1 \mathrm{~g} / \mathrm{L})$ as the base medium. After autoclaving the solution $\left(121^{\circ} \mathrm{C}, 15\right.$ pounds for $\left.15 \mathrm{~min}\right)$, horse serum (100cc), bacitracin $(75 \mu \mathrm{g} / \mathrm{ml})$ and vancomycin $(2.5 \mu \mathrm{g} / \mathrm{ml})$ were added to the medium when it reached $45^{\circ} \mathrm{C}$ temperature. The medium was then dispensed into microbiological plates.

In the laboratory, the paper cones in the center of the plates were rolled to the edges with a sterile microbiological loop to impregnate the microorganisms and then set in an anaerobic jar (with a gaspack). The jar was kept in an incubator for 5 days.

After 5 days of incubation at $37^{\circ} \mathrm{C}$, the plates were evaluated for detection of $A$. actinomycetemcomitans colony by a light microscope with $10 \mathrm{X}$ lens and star-shaped $A$. actinomycetemcomitans colonies were counted.

\section{RESULTS}

All the 24 patients enrolled into the study completed all examinations throughout the 6 months study period. None of the patients reported adverse effects due to taking the antibiotics. The baseline examinations data in test and control patients are shown in Table 1.

As indicated in Table 1, differences between test and control patients in all parameters are inconsiderable. Follow-up examinations took place 10 days, 3 and 6 months after the mechanical treatment.

As shown in Table 2, which is the static outcome of clinical attachment loss data in test and control patients, there was no significant difference between test and control groups at any stage $(P>0.05)$. Table 3 shows the $A$. actinomycetemcomitans colony count of patients in each group in all research stages. Significant differences 
Table 1. Assessed parameters in patients with A. actinomycetemcomitans-associated periodontitis before undergoing metronidazole-ciprofloxacin-therapy.

\begin{tabular}{|c|c|c|c|}
\hline Parameter & Test & Control & P-values \\
\hline Mean CC & 21.29 & 21.66 & 0.956 \\
\hline Mean CAL & 4.58 & 4.58 & 0.942 \\
\hline Mean PI & 96.9 & 95.1 & 0.747 \\
\hline $\mathrm{BOP}+$ & $12(100 \%)$ & \multicolumn{2}{|c|}{$12(100 \%)$} \\
\hline Mean GI & 2 & \multicolumn{2}{|c|}{2} \\
\hline
\end{tabular}

CC: Colony count (colonies of $A$. actinomycetemcomitans seen under microscope), CAL: clinical attachment loss (measured by use of periodontal probe), PI: plaque index (percentage of teeth surfaces with plaque disclose by plaque indicator tablets), BOP: bleeding on probing, Gl: gingival index (Loe and Silness, 1963).

Table 2. Mean clinical attachment loss in test and control patients at different stages of the research.

\begin{tabular}{lccc}
\hline Parameter & Test & Control & P-value \\
\hline Baseline & 4.58 & 4.58 & 0.942 \\
10 Days & 3.26 & 3.27 & 0.944 \\
3 Months & 3.27 & 3.07 & 0.539 \\
6 Months & 3.27 & 3.08 & 0.571 \\
\hline
\end{tabular}

Table 3. A. actinomycetemcomitans colony count of test and control patients at different stages of research.

\begin{tabular}{llcccc}
\hline Parameter & Group & Min. & Max. & Mean & P-value \\
\hline \multirow{2}{*}{ Baseline } & Test & 3.5 & 54.75 & 21.23 & \multirow{2}{*}{0.956} \\
& Control & 3.75 & 55.5 & 21.67 & \\
\multirow{3}{*}{10 days } & Test & 0 & 2.75 & 0.35 & \multirow{2}{*}{0.086} \\
& Control & 0 & 21.25 & 4.04 & \\
\multirow{3}{*}{3 months } & Test & 0 & 3.25 & 0.75 & \multirow{2}{*}{0.022} \\
& Control & 1 & 32.5 & 9.15 & \\
& Test & 0 & 7.5 & 1.96 & \multirow{2}{*}{0.008} \\
\hline
\end{tabular}

were seen at 3rd and 4th examinations (months 3 and 6) between test and control groups $(P<0.05)$.

Patients bleeding on probing are shown in Table 4. Again, at $3 \mathrm{rd}$ and 4th examinations, significant differences were seen between test and control group $(P<0.05)$. Test and control group showed significant differences at 3rd and 4th examinations in gingival index $(P<0.05)$. Table 5 demonstrates gingival indices of test and control patients. In Table 6, plaque indices of patients are shown. No significant difference was seen at any stage of research between test and control group $(P>0.05)$.

\section{DISCUSSION}

The aim of the present study was to evaluate the longterm microbiological and clinical effects of mechanical debridement, followed by ciprofloxacin-metronidazole therapy in $A$. actinomycetemcomitans-associated periodontitis. The results show that this combined therapy is effective in suppressing $A$. actinomycetemcomitans below cultivable levels in periodontal pocket, and also in treatment of bleeding on probing and high gingival index. No significant difference was seen in plaque index and attachment loss between test and control groups.

A. actinomycetemcomitans was suppressed below cultivable level in 21 out of 24 patients within the first 10 days of the study, while 9 patients in the control group still had $A$. actinomycetemcomitans in their periodontal pockets. Although, this result did not show significant difference, significant difference was found at 3rd and 6th months of the study.

Similar results have been reported by Muller et al. (1998), Soleymani et al. (2004), Yek et al. (2010), Aimetti et al. (2012), Heller et al., (2011), Soleymani et al. (2004), 
Table 4. Number of patients with bleeding on probing in different research stages.

\begin{tabular}{llcccccc}
\hline Parameter & Group & No tooth & One tooth & Two teeth & Three teeth & Four teeth & P-value \\
\hline \multirow{2}{*}{ Baseline } & Test & - & - & - & - & 12 & - \\
& Control & - & - & - & - & 12 & \\
\multirow{5}{*}{10 Days } & Test & 12 & - & - & - & - & 0.319 \\
& Control & 9 & 3 & - & - & - & 0.000 \\
\multirow{5}{*}{3 Months } & Test & 12 & - & - & - & - & \\
& Control & 2 & 5 & 4 & 1 & - & - \\
\multirow{2}{*}{6 Months } & Test & 12 & - & - & - & - & 0.000 \\
\hline
\end{tabular}

Table 5. Gingival indices of test and control patients in different research stages.

\begin{tabular}{llcccc}
\hline Parameter & Group & Min. & Max. & Mean & P-value \\
\hline \multirow{2}{*}{10 Days } & Test & 0 & 0.25 & 0.04 & \multirow{2}{*}{0.101} \\
& Control & 0 & 1.25 & 0.38 & \\
\multirow{3}{*}{3 Months } & Test & 0 & 0.25 & 0.06 & \multirow{2}{*}{0.000} \\
& Control & 0.25 & 1.75 & 1.02 & \\
& & & & & \multirow{2}{*}{6 Months } \\
& Test & 0 & 0.25 & 0.10 & 0.000 \\
\hline
\end{tabular}

Table 6. Plaque indices of test and control patients at different research stages.

\begin{tabular}{lccc}
\hline Parameter & Test & Control & P-value \\
\hline Baseline & 96.92 & 95 & 0.747 \\
10 Days & 43.58 & 37.33 & 0.414 \\
3 Months & 69.33 & 70.17 & 0.871 \\
6 Months & 81.33 & 81.58 & 0.964 \\
\hline
\end{tabular}

be considered.

This treatment was also able to decrease bleeding on probing by significant difference between test and control groups in our study. None of the patients in test group had bleeding on probing at any of the research stages.

Lopez et al. (2000), Yek et al. (2010), Aimetti et al. (2012) and Heller et al., (2011) have reported similar results. Adverse result was reported by Bain et al. (1994), since they were unable to show significant difference in bleeding on probing between test and control groups, 24 weeks after the treatment. Different medication seems to be the cause of this controversy, as in Bain's (1994) research, the patients received spiramycin 1.5 million IU bid, and in this study, the patients received ciprofloxacin $500 \mathrm{mg}$ bid plus metronidazole $250 \mathrm{mg}$ tid.

In this study, no significant differences were seen in attachment loss between two groups. Tezel et al. (2005) demonstrated significant differences in attachment loss between test and control groups at the 3rd month of their study. They used different product of ciprofloxacin (Siprosan $500 \mathrm{mg}$ ) and in higher dose (tid) and this seems to be the cause of this controversy. Flemmig et al. (1998) also showed significant difference in attachments loss between test and control groups at 12th month of their study. Such controversy may be the result of different attachment loss measurement devices usage. In this study, no significant difference was seen in plaque indices between test and control groups at any stages of the study.

Tinoco et al. (1998) and Bain et al. (1994) were unable to show any significant difference in plaque index between test and control groups, which is similar to the 
results of this study. According to the results of this study, we conclude that systemic application of ciprofloxacin plus metronidazole adjacent to mechanical debridement is effective for suppression of $A$. actinomycetemcomitans and clinical improvement of periodontal tissues.

\section{ACKNOWLEGEMENTS}

The authors are grateful to Dr. Moubedi and Dr. Nateghi for providing the antibiotics and placebos with no charge. They are also thankful to the Microbiology Group of Iran University of Medical Sciences, especially Mr. Masjedian for providing the laboratory facilities.

\section{ABBREVIATIONS}

${ }^{\circ} \mathbf{C}$, Degree Celsius; $\boldsymbol{\mu g}$, microgram; A.a, actinobacillus actinomycetemcomitans; att., attachment; bid, bis in die (two times a day); BOP, bleeding on probing; CAL, clinical attachment loss; cc, cubic centimeter (milliliter); GI, gilgival index; g, gram; IU, international unit; mg, milligram; $\mathbf{m l}$, milliliter; $\mathbf{m m}$, millimeter; NSAID, nonsteroid anti-inflammatory drug; $\mathbf{P}$, probability value $(p-$ value); PI, plaque index; S/RP, scaling and root planing; tid, ter in die (three times a day); TSBV, tryptic soy serum plus bacitracin and vancomycin.

\section{Competing Interests}

The authors hereby declare that there were no competing interests.

\section{REFERENCES}

Aimetti M, Romano F, Guzzi N, Carnevale G (2012). Full-mouth disinfection and systemic antimicrobial therapy in generalized aggressive periodontitis: a randomized, placebo-controlled trial. J. Clin. Periodontol. 39(3):284-294.

Bain CA, Beagrie GS, Bourgoin J, Delorme F, Holthuis A, Landry RG, Roy S, Schuller P, Singer D, Turnbull R (1994). The effects of spiramycin and/or scaling on advanced periodontitis in humans. J. Can. Dent. Assoc. 60:209-217.

Flemmig TF, Milian E, Karch H, Klaiber B (1998). Differential clinical treatment outcome after systemic Metronidazole and amoxicillin in patients harboring Actinobacillus actinomycetemcomitans and/or Prophyromonas gingivalis. J. Clin. Periodontol. 25:380-387.
Heller D, Varela VM, Silva-Senem MX, Torres MC, Feres-Filho EJ, Colombo AP (2011). Impact of systemic antimicrobials combined with anti-infective mechanical debridement on the microbiota of generalized aggressive periodontitis: a 6-month RCT. J. Clin. Periodontol. 38(4):355-364.

Karen FN, Novak MJ (2006). Aggressive Periodontitis. In: Newman MG, Takei HH, Klokkevold PR, Carranza FA (eds.), Carranza's Clinical Periodontology, 10th Edition. W.B. Saunders. Chapter 33, Page 507.Lopez NJ, Gamonal JA, Martinez B (1998). Repeated metornidazole and amoxicillin in the treatment of periodontitis. A follow up study. J. Periodontol. 71:79-89.

Marc Q, Wim T, Susan K, Haake T, Michael GN (2006). Microbiology of Periodontal Diseases. In: Newman MG, Takei HH, Klokkevold PR, Carranza FA (eds.), Carranza's Clinical Periodontology, 10th Edition. W.B. Saunders. Chapter 9, pp 137-161.

Müller HP1, Heinecke A, Borneff M, Kiencke C, Knopf A, Pohl S (1998). Eradication of Actinobacillus actinomycetemcomitans from the oral cavity in adult periodotitis. J. Periodontal Res. Jan; 33(1):49-58.

Pavicić MJ, van Winkelhoff AJ, Douqué NH, Steures RW, de Graaff J. (1994). Microbiological and clinical effects of Metronidazole and amoxicillin in Actinobacillus actinomycetemcomitans-associated periodontits- A 2-year evaluation. J. Clin. Periodontol. 21:107-112.

Renvert S, Wikström M, Dahlén G, Slots J, Egelberg J (1990). Effect of root debridement on the elimination of Actinobacillus actinomycetemcomitans and Bacteroides gingivalis from periodontal pockets. J. Clin. Periodontol. 17(6):345-350.

Slots $J$ (2002). Selection of antimicrobial agents in periodontal therapy. J. Periodont. Res. 37:389-398.

Soleymani SY, Khorsand A, Salary MH, Mehrizy H (2004). Comparison of systemic Ciprofloxacin in elimination of Actinobacillus actinomycetemcomitans from active sites with combination of Metronidazole and Amoxicillin in patients with aggressive periodontitis. A randomized double blind controlled trial. J. Dentistry Tehran Univ. Med. Sci. 1(2):24-28.

Tezel A, Yucel O, Orbak R, Kara C, Kavrut F, Yagiz H, Sahin T (2005). The gingival cervicular fluid ciprofloxacin level in subjects with gingivitis and periodontitis, and its effect on clinical parameters. J. Periodont. Res. 40:395-400.

Tinoco EM, Beldi MI, Campedelli F, Lana M, Loureiro CA, Bellini HT, Rams TE, Tinoco NM, Gjermo P, Preus HR (1998). Clinical and microbiological effects of adjunctive antibiotics in treatment of localized juvenile periodontitis. A controlled clinical trial. J. Periodontol. 69(12):1355-1363.

Van Winkelhoff AJ, Carolien J (1992). Microbiological and clinical results of Metronidazole plus Amoxicillin therapy in A.a associated periodontitis. J. Periodontol. 63:52-57.

Yek EC, Cintan S, Topcuoglu N, Kulekci G, Issever H, Kantarci A (2010). Efficacy of amoxicillin and metronidazole combination for the management of generalized aggressive periodontitis. J. Periodontol. 81(7):964-974 